\title{
Cryogenic optical performance of the Cassini Composite InfraRed Spectrometer (CIRS) flight telescope
}

\author{
Patricia Losch, James J. Lyons III, John Hagopian \\ Optics Branch Code 551 \\ NASAVGoddard Space Flight Center \\ Greenbelt, MD 20771
}

\begin{abstract}
The CIRS half-meter diameter beryllium flight telescope's optical performance was tested at the instrument operating temperature of 170 Kelvin. The telescope components were designed at Goddard Space Flight Center (GSFC) but fabricated out of house and then assembled, aligned and tested upon receipt at GSFC. A 24 inch aperture cryogenic test facility utilizing a 1024 × 1024 CCD array was developed at GSFC specifically for this test. The telescope's image quality (measured as encircled energy), boresight stability and focus stability were measured. The gold coated beryllium design exceeded the cold image performance requirement of $80 \%$ encircled energy within a 460 micron diameter circle.

Keywords: cryogenic, optical test, encircled energy, beryllium cassegrain telescope
\end{abstract}

\subsection{Introduction}

The CIRS is a remote sensor built as an international collaboration at the Goddard Space Flight Center'. CIRS was launched on NASA's Cassini Orbiter to Saturn in October 1997. Infrared spectroscopy of emissions from Saturn's and Titan's atmospheres, rings, and surfaces will provide 3-D and temporal variations of temperature distribution, gas abundances, aerosols, and clouds. Global mapping of Saturn's rings and icy satellite for composition and thermal properties will also be performed. ${ }^{2}$

CIRS consists of a 0.5 meter F/6 Cassegrain beryllium telescope, a reference interferometer to provide scan mechanism control and timing for data sampling, and two science Fourier transform spectrometers: a MidInfrared interferometer and a Far infrared interferometer. The MIR is a classical Michelson interferometer with a 7 - 17 micron spectral range measured with two linear $\mathrm{HgCdTe}$ arrays (FP3 and FP4) consisting of ten 200 micron square pixels each viewing 0.273 mrad field. The FIR is a polarizing Michelson interferometer measuring from $17-1000$ microns using two $4.3 \mathrm{mrad}$ thermopile detectors (FP1). The CIRS fields of view are shown in Figure 1.

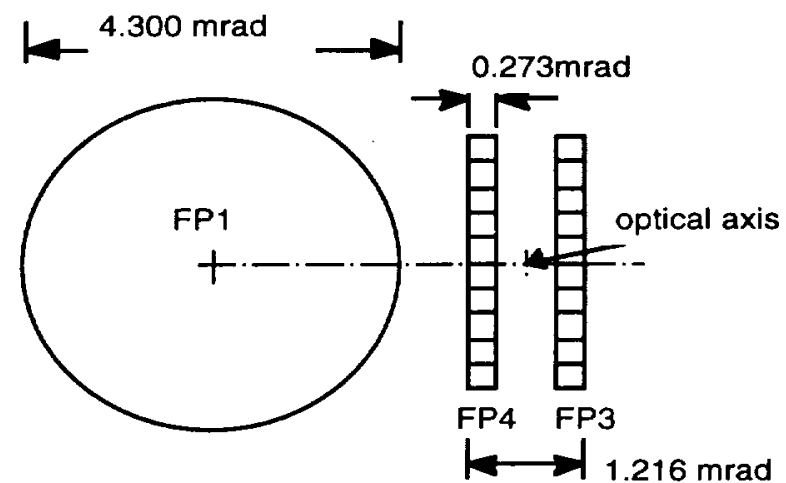

Figure 1. CIRS fields of view consisting of focal planes 1 (FIR), 3, and 4 (MIR)

CIRS is a passively cooled cryogenic instrument. All subsystems with the exception of the MIR focal plane operate at $170 \mathrm{Kelvin}$. The MIR focal plane operates at $80 \mathrm{Kelvin}$ and is cooled using a honeycomb radiative patch. 


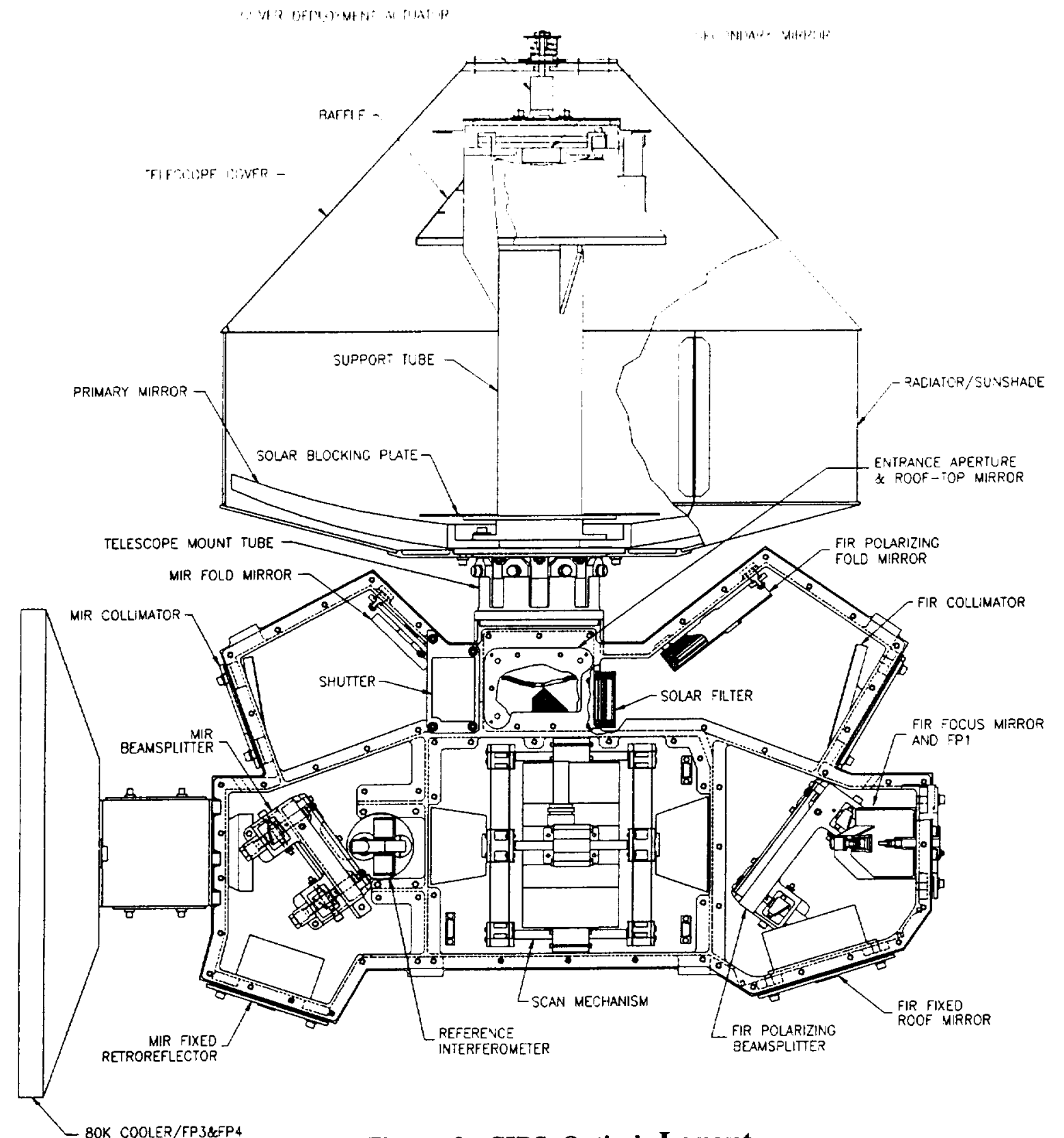

Figure 2: CIRS Optical Layout

The optical design of CIRS is described by Maymon $\mathrm{et}^{\mathrm{al}^{3}}$. A brief description of the optical layout of CIRS shown in Figure 2 is presented here. Light is collected across the 12.426 mrad field of view by the 0.5 meter, F/6 Cassegrain telescope. The beryllium telescope is mounted via a stress-free transition tube to the aluminum optics bench which houses the collimating optics and interferometers. A parabolic Entrance Aperture Plate (EAP) at the telescope focal surface serves to block stray light and is mounted in front of and to the field-split mirror. The EAP contains two apertures coincident with the MIR and FIR fields of view. The MIR and FIR fields of view are divided by the field split mirror which consists of two weak spherical mirrors each at $45^{\circ}$ from the telescope optical axis. The FIR surface of the field split mirror was roughened and works in conjunction with a solar blocking filter to prevent solar radiation from traveling through the FIR interferometer and damaging the thermopile detectors. Both the MIR and FIR beams are then collimated by off-axis parabolas and steered into the interferometers via a fold flat in the MIR and an input polarizer for the FIR. The MIR 
contains a Potassium Bromide beamsplitter and compensator. The FIR utilizes a polarizing grid identical to the input polarizer (but rotated $45^{\circ}$ ) as its beamsplitter. The tixed and moving mirrors are not typical flat mirrors but are cube corners in the MIR and dihedral mirrors in the FIR. These were developed at GSFC ${ }^{3}$ to provide relief in the performance requirements of the mirror scanning mechanism. The dihedrals also serve to rotate the beams' polarizations. After passing through the fixed and moving legs of interferometer the MIR beam is focused onto the detector arrays by an F/l germanium lens. The FIR beam is focused by an F/l on-axis parabola through an output analyzer and onto the corresponding thermopile detectort. Both signals are then Fourier transformed to provide spectral information.

\subsection{Telescope History}

Originally the CIRS project had baselined the use of one of two existing spare telescopes from the Voyageur Mid-Infrared Interferometer Spectrometer (MIRIS) as the CIRS flight unit. Extensive testing was performed by GSFC in conjunction with Applied Optics Associates and ITEK on both the primary mirrors and the telescopes. However, the MIRIS telescopes' cold performances did not satisfy the CIRS required cold encircled energy of $80 \%$ in a 460 micron spot diameter. The warm performance of the best of the two units was $80 \%$ in 880 microns. The spot size grew to $80 \%$ in 2200 microns at the operating temperature of $170 \mathrm{Kelvin}$ ?. This was the first cryogenic image quality data obtained for the MIRIS units. Previously, the telescopes were simply cooled to $135 \mathrm{Kelvin}$ and the absence of change in the warm image spot size was used as performance acceptance criteria ${ }^{8}$.

The degraded telescope performance at cold temperatures is attributed almost entirely to the primary mirror. The contributing factors include anisotropy of the 170 vacuum hot pressed (VHP) beryllium, bimetallic stresses from the nickel plating, uneven nickel thickness, and an asymmetric lightweighting design. The anisotropy of the 170 VHP beryllium ranges is approximately $4 \%$. The nickel thickness was estimated (based on a third unit measured and used on the Mars Observer Laser Altimeter project) to vary from 64 to 97 micorns ( 0.0025 to 0.0038 inches) on the front and 122 to 147 microns ( 0.0048 to 0.0058 inches) on the back. The MIRIS lightweighting pattern is shown in Figure 3. 
Having determined that the MIRIS telescopes could not be used for hight, a new telescope was baselined. (One of the existing MIRIS units was used for the CIRS engineering model.) Emphasis was placed on redesigning the primary mirror.

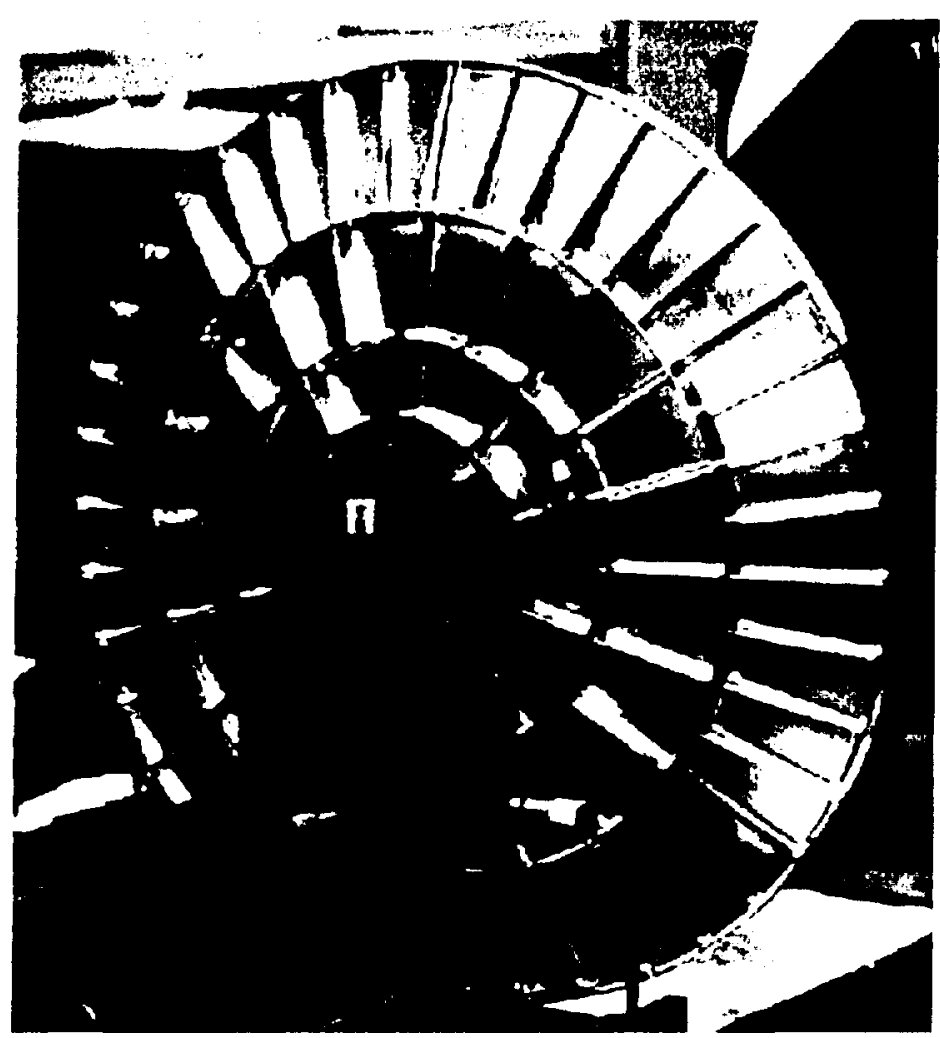

Figure 3: MIRIS Primary Mirror Lightweighting Design

\subsection{Telescope Design}

For the CIRS flight telescope, we retained the same optical prescription as the MIRIS unit but redesigned the opto-mechanical properties of the telescope. In order to minimize cost, the redesign was performed at GSFC, the fabrication was contracted out with the final assembly and cryogenic image quality tests done at GSFC in a test facility built for this effort. We felt that by eliminating the factors contributing to the distortion of the primary mirror at $170 \mathrm{Kelvin}$ we could, with low risk, accept delivery of components built to spec, cold test the assembly in-housc, and have a telescope which met our requirements.

The optical prescription was that of a 3 meter focal length Cassegrain design and consisted of a parabolic primary with a vertex focal length of $35.54 \mathrm{~cm}$ ( 14 inches), outside clear aperture of 0.508 meters (20 inches) and an inside clear aperture of $14.22 \mathrm{~cm}$ ( 5.6 inches). The secondary mirror is a convex hyperboloid having a vertex radius of curvature of $11.5 \mathrm{~cm}$ (4.528 inches) and an outside clear aperture diameter of 7.96 $\mathrm{cm}$ (3.133 inches) and an inside clear aperture of $2.54 \mathrm{~cm}$ ( 1.0 inch). Both mirrors had a $40 \AA \mathrm{rms}$ microroughness requirement. The lelescope assembly initially had a back focial length requirement of $11.476 \mathrm{~cm} \pm .05 \mathrm{l} \mathrm{cm}(4.518 \pm .020$ inches) but this was modified to $11.605 \mathrm{~cm}-0.013+0.025 \mathrm{~cm}(4.569$ $-0.005+0.010$ inches) based on as-built alignment and test data of the collimating optics". The boresight 
requirement wats that the blur circle center be located within a 18.3 micron (0). $(0) 72$ inch) ratius of the primary mirror eptical axis.

The telescope design utilizes the newer 170 hot isostatic pressed (HIP) beryllium. symmetric lightweighting design, and hare beryllium polishing and coating technology. The HIP beryllium has $0.4 \%$ anisotropy compared with $4 \%$ for the 170 VHP beryllium. The lightweighting paltern designed by Bill Hayden and Pam Generie of GFSC is shown in Figure 4 and shows the tri-cell symmetry which is not present in the MIRIS unit. Finally, the bimetallic effects were eliminated by utilizing new capabilities in bare beryllium polishing and protected, evaporated gold coating..

$$
\text { Ser a thichel }
$$

\section{Figure 4: CIRS Primary Mirror Lightweighting Design}

The secondary mirror did not undergo redesign but was fabricated out of the new $170 \mathrm{H}$ optical grade beryllium. The mirror was then nickel plated with an overcoat of gold plating.

All the beryllium was purchased from Brush-Wellman and machined by Speedring. The primary mirror was ground and polished by OCA Applied Optics and coated by Denton Vacuum. The secondary mirror was also ground and polished by OCA who also applied the electroless nickel plating. The final gold plating was done at GSFC.

An integral part of the telescope assembly used for both the engineering and thight unit telescopes is the transition tube which provides a stress free interface between the beryllium primary mirror and the aluminum optical bench.

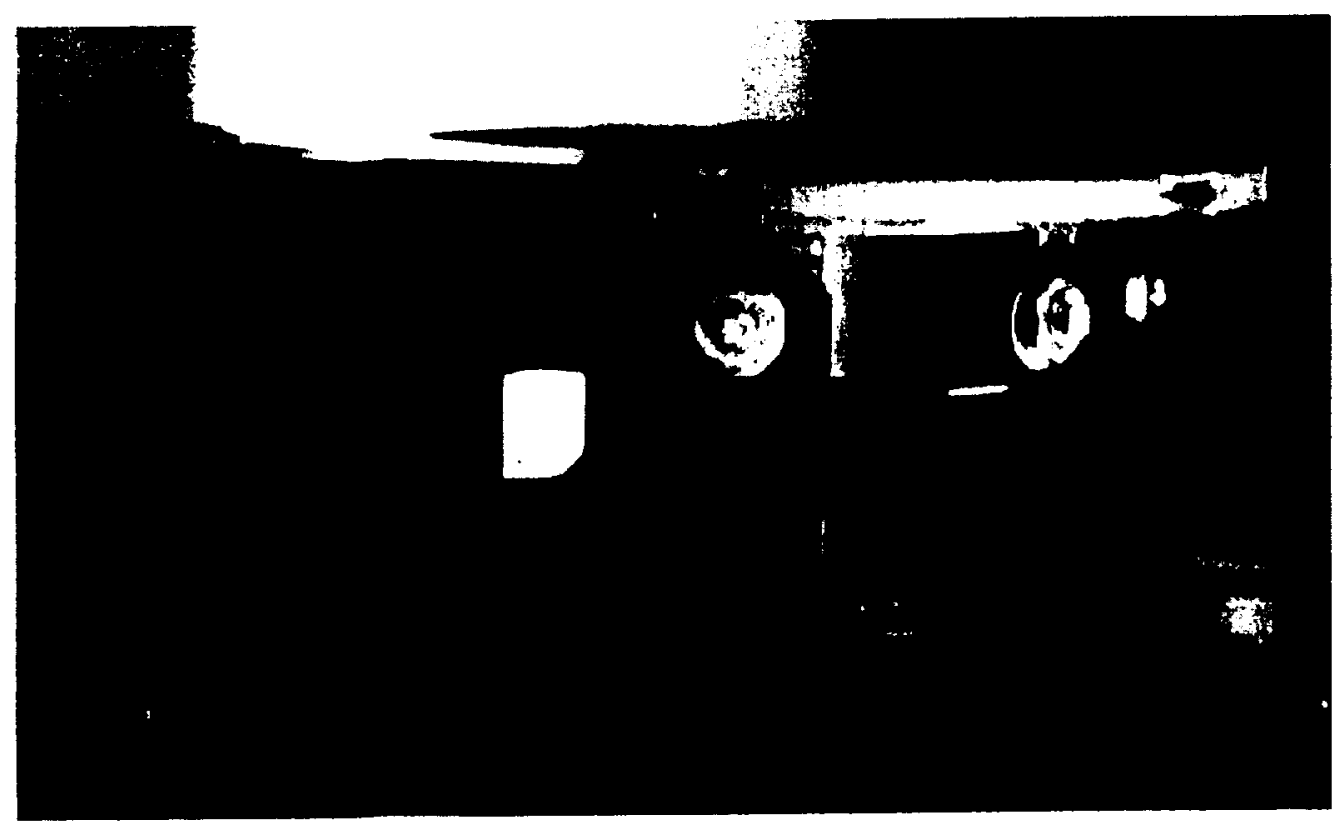

Figure 5: CIRS Aluminum to Beryllium Transition Tube

\subsection{Warm Assembly and Alignment}

The telescope assembly was received as individual components: the primary mirror (PM), secondary mirror (SM), the secondary mirror support tube (SMST), secondary mirror shims, and transition tube. The parts 
were assembled, aligned, and lested at room temperature, then integrated into the cold chamber for testing at $170 \mathrm{~K}$.

Upon delivery and assembly of the telescope components, we first measured the back focal position of the system. A set of secondary mirror shims were located between the secondary mirror support tube and the secondary mirror mount. These beryllium shims were lapped to adjust the back focal length of the telescope.

The transition tube, primary mirror, and the SMST were first bolted together via eight bolts around the center of the primary mirror. A beryllium centering sleeve which rested in the transition tube was used to center the SMST onto the transition tube. This assembly was mounted onto a one inch thick aluminum plate polished flat on both sides with the surfaces parallel. The assembly was then mounted on a gimbal mount in front of a 24 inch collimated helium-neon beam.

We now aligned the primary mirror optical axis to the collimated beam as shown in Figure 6. To do this, we placed a theodolite behind the primary mirror looking through its central hole at the collimated beam. A crosshair reticle was then installed into the primary mirror's central hole and the theodolite was translated and tilted until we could scan through its focus to view the crosshairs and the image formed by the primary mirror on the same axis. The theodolite was then focused at infinity to view the axis of the collimated beam. The primary mirror assembly was now tilted to place its optical axis coincident with the collimated beam. When this occurred, the focus of the theodolite could be adjusted and the image, the crosshairs and the collimated beam would all lie along the same direction. To verify that the image and the crosshairs defined the PM optical axis, a fiber light was placed at the on-axis radius of curvature such that the light was returned back on itself. This was observed to be on the same axis as that defined by the center of the PM central hole and the PM image of the collimated beam.

Figure 6: Alignment of PM optical axis See A Ha ched As an alignment aid, we then characterized the pointing direction of the aluminum interface plate with respect to the PM optical axis. This gave the offset of the transition tube interface to the optical axis of the PM which was 15 arc seconds in azimuth (horizontal) and 8 arc seconds in elevation (vertical). We could then easily refer to the flange pointing direction to align the PM to the collimated beam without going through the cumbersome procedure described in the preceding paragraph.

The secondary mirror and shims were then installed and the back focal position was measured. The SM decenter position was first adjusted to minimize the image size and off axis misalignment as viewed through a microscope behind the telescope. The microscope was mounted on a three-axis translator and a reticle was mounted to the transition tube as shown in Figure 7. Two different reticles were used. The first reticle was used to measure decenter. This reticle was on a glass plate precisely centered with respect to the central hole of the primary mirror. We first focused the microscope on the center of the reticle and its horizontal and vertical micrometer readings were recorded. We then translated the microscope to the center of the image. The difference between the two sets of micrometer readings was the boresight error. The glass reticle was then replaced with a thin transparency whose focus position with respect to the back surface of the primary mirror had been well characterized. Again, we first focused the microscope on the transparency and translated back to focus on the image. These two focus positions gave us the back focal distance (measured from the back flange of primary mirror). The focus error was divided by the longitudinal magnification of 73.44 to determine the amount of material to remove from the secondary mirror shims. 


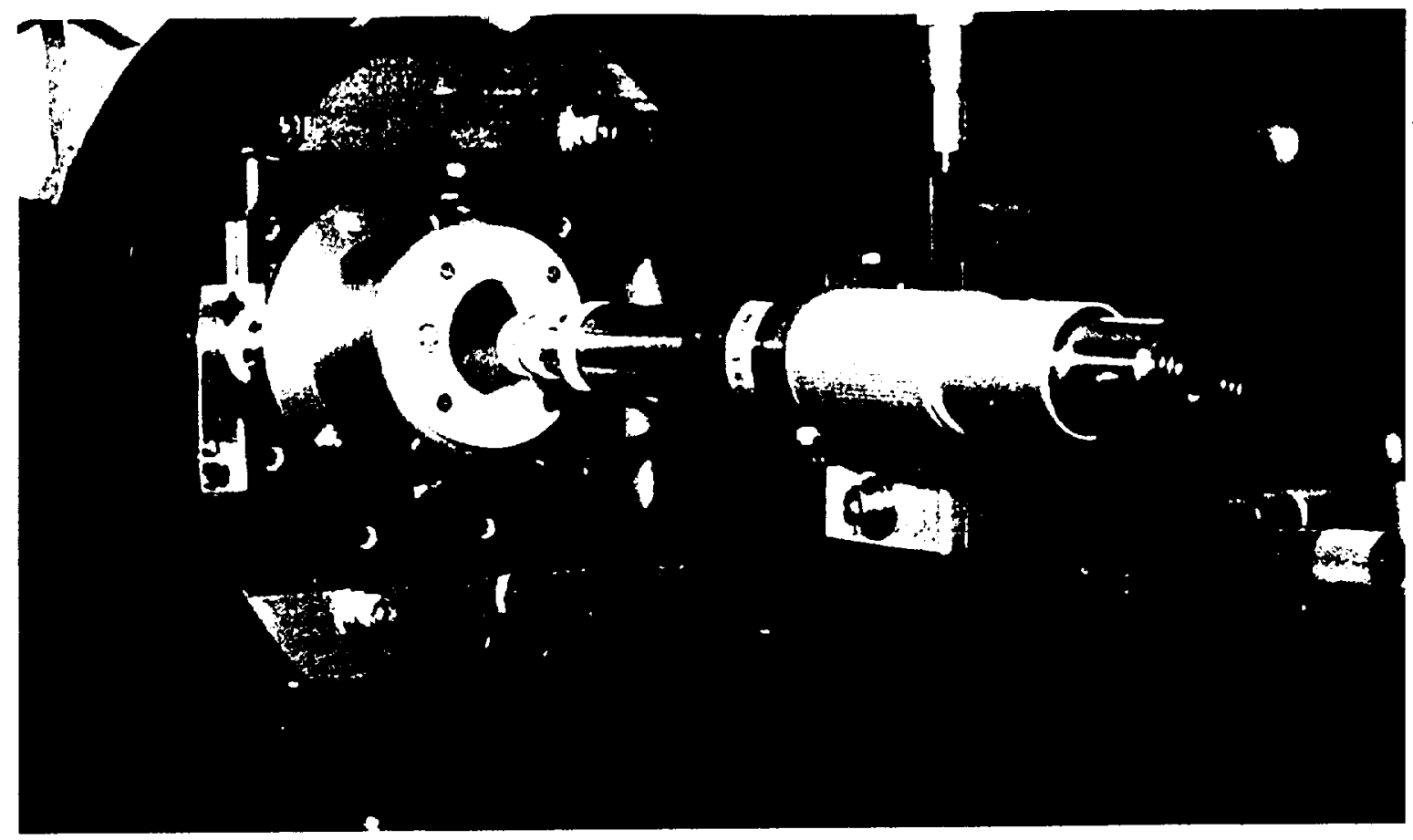

Figure 7: Measurement of back focal position

The SM shims were gradually lapped (removing only 2.5 to 5 microns each time) to slowly bring the image to within the envelope for the back focal distance. When we achieved a back focal length of 11.615 $\mathrm{cm}$ (4.573 inches), we measured the boresight error and image size, also using the microscope. The $100 \%$ image size was approximately 430 microns in diameter and was measured by translating the microscope from one edge of the image to the other.

The boresight error measured was 48 arcseconds in elevation $(0.69 \mathrm{~mm}$ vertical decenter down) and 12 arcseconds in azimuth $(0.18 \mathrm{~mm}$ horizontal decenter to the right). The vertical boresight error value exceeded the boresight requirement that the image fall within $0.183 \mathrm{~mm}$ of the optical axis. To accommodate this boresight error we first verified that tilting the telescope by the above amounts placed the image on axis. We then verified that no noticeable change in image resulted. With this information we were able to overcome the boresight error. We did this by tilting the CIRS instrument, after the telescope was integrated, to center the telescope image on the MIR field and shimming the focal planes to eliminate any vignetting.

To assist our alignment of the telescope to the collimated beam once the telescope had been secured in the cold chamber, we characterized the pointing direction of the back of the SM to the telescope optical axis. This offset value was 15 arcseconds in azimuth and 4 arcseconds in elevation. The assembled telescope is shown in Figure 8 without harnessing and blankets. 


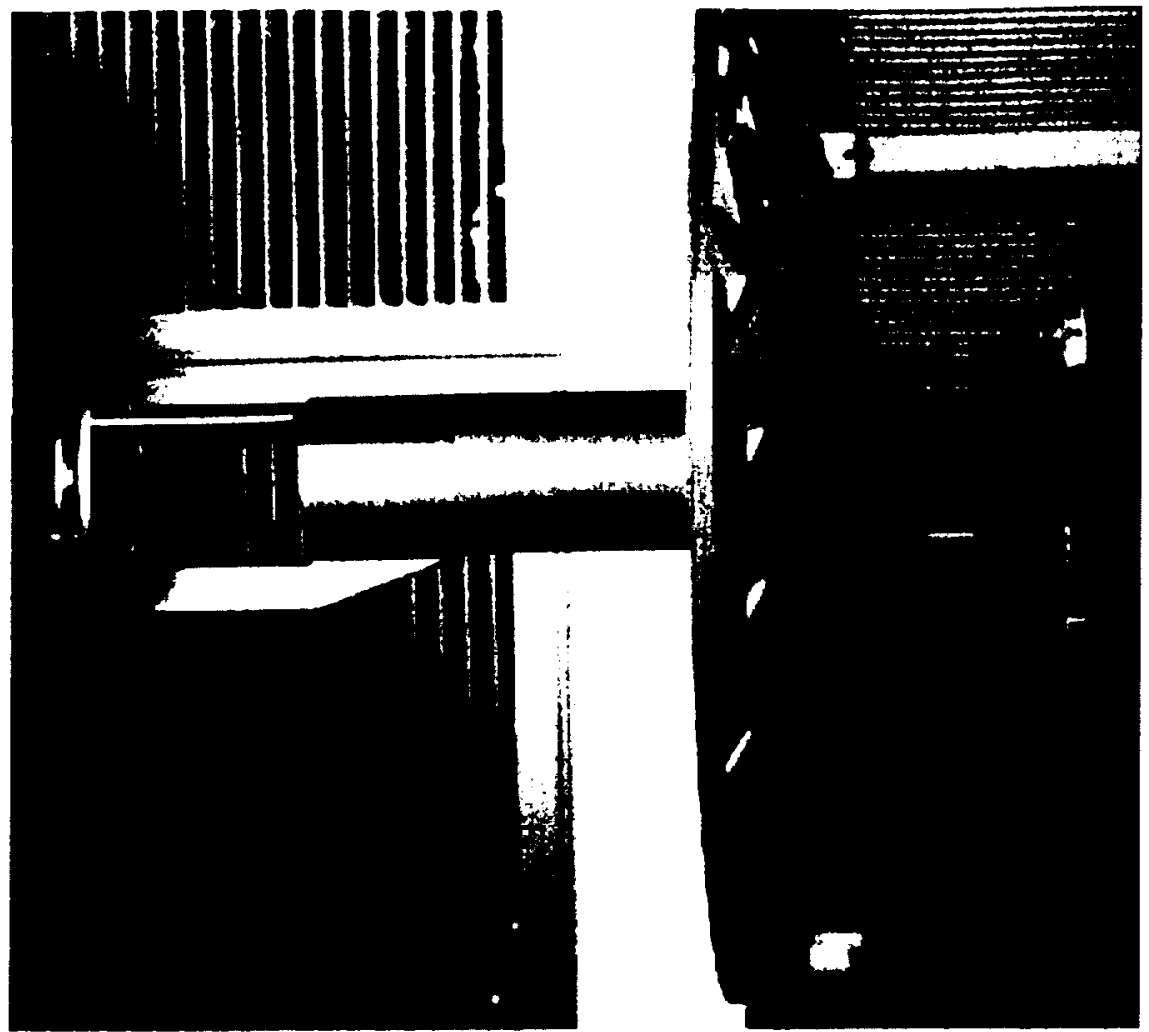

Figure 8: Side view of the assembled telescope

\subsection{Cryogenic Test Chamber}

To additionally reduce the cost of the flight telescope, the cryogenic optical performance verification testing was brought in-house rather than contracted out. The facility, developed specifically for this test, utilized a chamber already on-hand from the Cosmic Background Explorer project. The chamber dimensions were modified to accommodate the telescope interface. We then integrated thermal instrumentation, shrouds and blanketing, CCD camera, and translation stages.

The chamber was originally designed for vertical use which would not suit our purposes. Therefore, we first built a dolly to mount it horizontally. The chamber's inside diameter of the nitrogen shroud was 0.86 meters ( 34 inche)s which easily accommodated the half-meter diameter telescope. However, an extension to the 1 meter inside length of the dewar was required. This extension was also 1 meter in length and was mounted on a separate dolly. A 0.61 meter $(24$ inch) diameter window was mounted in the extension as were two feedthroughs for the tip and tilt alignment rods. Figure 9 shows the outside configuration of the modified chamber.

$$
\text { sed attided }
$$

Figure 9: Cryogenic Chamber

The telescope interface was via a second polished aluminum plate which also bolted onto the transition tube. This plate contained copper tubing through which liquid nitrogen flowed and cooled the telescope assembly. The telescope is then bolted via the cold plate onto a gimbal mount which connected to the tip and tilt rods. The gimbal mount is secured to a large platform on rails and which can roll out of the chamber onto a mating cart allowing easy installation and handling.

A 24 inch diameter, 22 inch long shroud painted thermally black was hard mounted to the large platform. The gimbal mount is positioned behind the shroud such that the SMST fits inside and the PM edge is flush with the end of the shroud as shown in Figure 10. The shroud provides radiative cooling of the SM and reduces thermal gradients. Multi-layer thermal blanketing material covers the back of the primary mirror and attaches to the shroud. This is required because the camera and translation stages are kept at ambient temperature. Platimun resistance thermometers are located on the back of the SM, along the length of the SMST and at multiple positions on the back of the primary mirror. $50 \mathrm{ohm}$ heaters were used in 
conjunction with a Lakeshore Cryotronics 330 Controller to maintain the $170 \mathrm{Kelvin}$ test temperature. A front view of the telescope installed in the chamber is shown in Figure 11.

$\left.\begin{array}{l}\text { Figure 10: The back of the primary mirror mounted onto the cold plate and gimbal } \\ \text { mount inside the shroud prior to attaching the thermal blanketing. } \\ \text { Figure 11: Front view of the telescope mounted in the chamber. }\end{array}\right\} a+f$ ched

A CCD camera was mounted on a three axis translation stage assembly which bolted to the large platform behind the gimbal mount. DynaOptic Motion motors were used to drive each translation stage. The motors were characterized to have less than 9 counts of backlash. Each motor was calibrated prior to the test. The focus motor has 1369 counts $/ \mathrm{mm}$ and the vertical and horizontal motors have 9690 counts $/ \mathrm{mm}$. The detector used for this test is a $1024 \times 1024$ array of 18 micron square pixels manufactured by Texas Instruments of Japan. It is a virtual phase charge coupled device running at $250 \mathrm{k}$ pixels per second with the drive electronics and software provided by the GSFC's Detector Systems Branch Code 553. Integration time is adjustable from 0.5 seconds to 1000 seconds and was limited by a large shutter used block the 4 inch collimated Zygo beam prior to the 24 inch beam expander. The camera, stages and motors are thermally isolated from the chamber and operate at ambient temperature and are shown in Figure 12.

\section{Figure 12: Side view of the CCD camera mounted on translation stages. CAtached.}

\subsection{Cryogenic Test}

After placing the back focal point at its correct position and characterizing the pointing direction of the telescope boresight with respect to the back flange of the secondary mirror, we integrated the telescope into the cold chamber.

Once assembled into the chamber and the chamber extension secured, the tip and tilt rods were used to align the telescope optical axis to the collimated beam. A theodolite looking at the return from the back of the SM was used to align the boresight of the telescope to the collimated beam to within 5 arcseconds. The test configuration is shown in Figure 13.

Figure 13: Alignment of the telescope to the collimated beam. A Huche d.

We were now ready to acquire ambient images. The first step in the image acquisition procedure was to darken the room, close the shutter, and record background image files. Each image saved is actually the average of four acquired frames. For each through focus series, a background image file was created and saved. This background file was specified as the reference frame which was automatically subtracted from each telescope image file. After obtaining the background image files, the shutter was activated and the camera was translated to the telescope focus and test images were acquired to check for saturation. The beam attenuation and shutter speed were adjusted accordingly. Several telescope images were acquired at motor increments of 600 counts ( 438 microns) over $\pm 2.36 \mathrm{~mm}$ range about focus.

A turbo pump was used to evacuate the chamber and the telescope was cooled to $170 \mathrm{Kelvin}$ at a rate of 20 degrees per hour as measured by the sensor located on the beryllium side of the transition tube. At this location, the temperature was consistently mid-way between that of the PM $(168 \mathrm{~K})$ and that of the SM $(172 \mathrm{~K})$. After the telescope has soaked for one hour, background and telescope image files are again acquired as described above.

In addition to verifying the cold encircled energy, the stability of the tclescope back focal point was verilied. First, the camera and telescope were removed and the chamber was instrumented with dial indicators on the translation stages with their points touching the telescope aluminum mounting plate. The readouts of the stages were recorded for specified indicator readings. Vacuum was pulled in the chamber and the mounting plate was cooled to 170 Kelvin. The stages were then moved out and back to repeat the indicator readings and the stage readouts recorded. The difference in stage counts provided us with the motion of the camera 
with respect to the telescope mounting plate between warm and cold. Secondly, we recorded the stage focus position readouts for best focus warm and cold These values were combined to give a net image motion with respect to the telescope interface.

The boresight stability was also verified. This was done detcrmining the centroid locations of the warm and cold images. The centroid positions could be directly compared because, prior to each set of through focus images, the telescope was aligned to the collimated beam.

\subsection{Results/Summary}

The encircled energy calculations were done after a full cycle of data was acquired. The encircled energy was calculated using a MatLab program. A threshold value was set below which the pixel would not be used in the sum. This was required because the camera exhibited a streaking both warm and cold which could not be contributed to saturation. The total energy was then summed, the centroid found and the $80 \%$ ensquared energy width about that centroid was calculated. (The $80 \%$ cold ensquared energy requirement was 500 microns.) These $80 \%$ values were then plotted as a function of focus position and the best focus position was determined. A near-best focus image for warm and cold are shown in Figure 14.

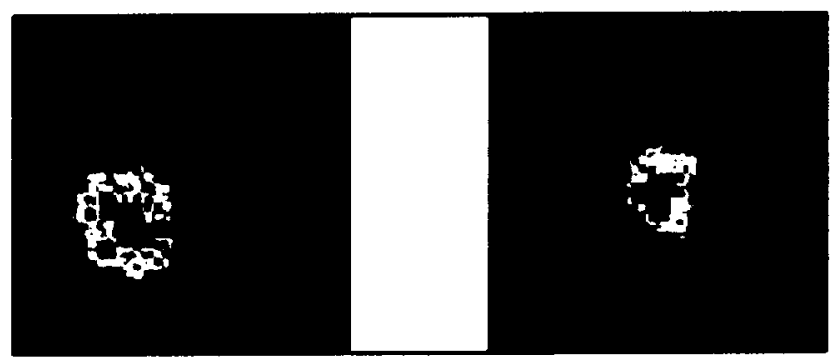

Figure 14: Near focus telescope images. Left is warm. Right is cold

The warm image size was 288 microns versus a cold image size of 207 microns. These measurements repeated to within \pm 1 pixel or \pm 18 microns.

The image movement in focus was determined to be 25 microns towards the telescope. This was calculated after measuring the camera to telescope separation increased by 127 microns between warm and cold. The camera had to move 152 microns closer to the telescope to be at the best focus at cold temperatures versus at warm. The cold image thus had a net motion of 25 microns closer to the telescope giving a final back focal length of 11.613 inches (nominal $=11.605-.013+.025 \mathrm{~cm}$ ).

As seen in the images in Figure 14, some boresight motion occurred. The image moved "up" 9 arcseconds ( 7 pixels) giving a final error of 39 arcseconds vertically (elevation). The image moved to the "left" on the CCD by 23 arcseconds ( 19 pixels) giving a final error of 35 arcseconds horizontally.

Both the image size and back focal position easily meet the required values at cold temperatures. As mentioned in section 4.0 the boresight error was removed by tilting the instrument to center the telescope image on the MIR field of view and then shimming the MIR focal plane assembly as needed.

\subsection{Acknowledgments}

The authors wish to thank Tom French, Lou Worell of NSIManTech, John Zaniewski, Dan McHugh, Armando Morell, Bill Hayden, Marilyn Kraut, Laddawan Ruamsuwan, Pam Generie, Ravi Kaipa of Hughes STX, Dan Musinski (formerly of NSI, now at Zygo Corp), and Swales Acrospace.

\subsection{References}


1. Kunde, V. et al., "Cassini infrared Fourier spectroscopic investigation", Cassini/Huygens: A mission to the Salurnian Systems, Vol. 2803, SPIE, Denver, CO, 1996.

2. Kunde, V. et al., "Infrared spectroscopic remote sensing from the Cassini orbiter", Optical Spectroscopic Instrumentation and Techniques for the 1990s, Vol. 1318, SPIE, Las Cruces, NM, 1990.

3. Maymon et al., "Optical design of the Composite Infrared Spectrometer (CIRS) for the Cassini Mission", Space Astronomical Telescopes and Instruments II, Vol.1945, SPIE, Orlando, FL, 1993.

4. Crooke, J.A. et al., "Flight qualification of the Cassini-Composite Infrared Spectrometer (CIRS) - Far Infrared (FIR) polarizing beamsplitter substrate: Mylar chosen over Polypropylene", Cryogenic Optical Systems and Instruments VII, Vol.2814, SPIE, Denver, CO, 1996.

5. Lyons III, James Joseph, and Patricia Ann Hayes. "High Quality Cryogenic Hollow Retroreflectors", Current Developments in Optical Design and Engineering V, Vol. 2540, SPIE, San Diego, CA, 1995.

6. Crooke, J.A. and John G. Hagopian. "Alignment and Cryogenic Testing of the Cassini-Composite Infrared Spectrometer (CIRS) Far Infrared (FIR) Focal Plane”, Cryogenic Optical Systems and Instruments VII, Vol.2814, SPIE,_Denver, CO, 1996.

7. Hayes, Patricia, Julie Crooke, Brendon Perkins. "Cryogenic and ambient testing of the CIRS beryllium telescopes", Cryogenic Optical Systems and Instruments VI, Vol.2227, SPIE, Orlando, FL, 1994.

8. Texas Instruments Drawing \#2017051, Dallas, TX 1976

9. Hayes, Patricia, James Lyons III, John Hagopian, "Alignment Verification by Wavefront Testing of the Composite Infrared Spectrometer", Cryogenic Optical Systems VII, Vol. 2814, SPIE, Denver, CO, 1996. 


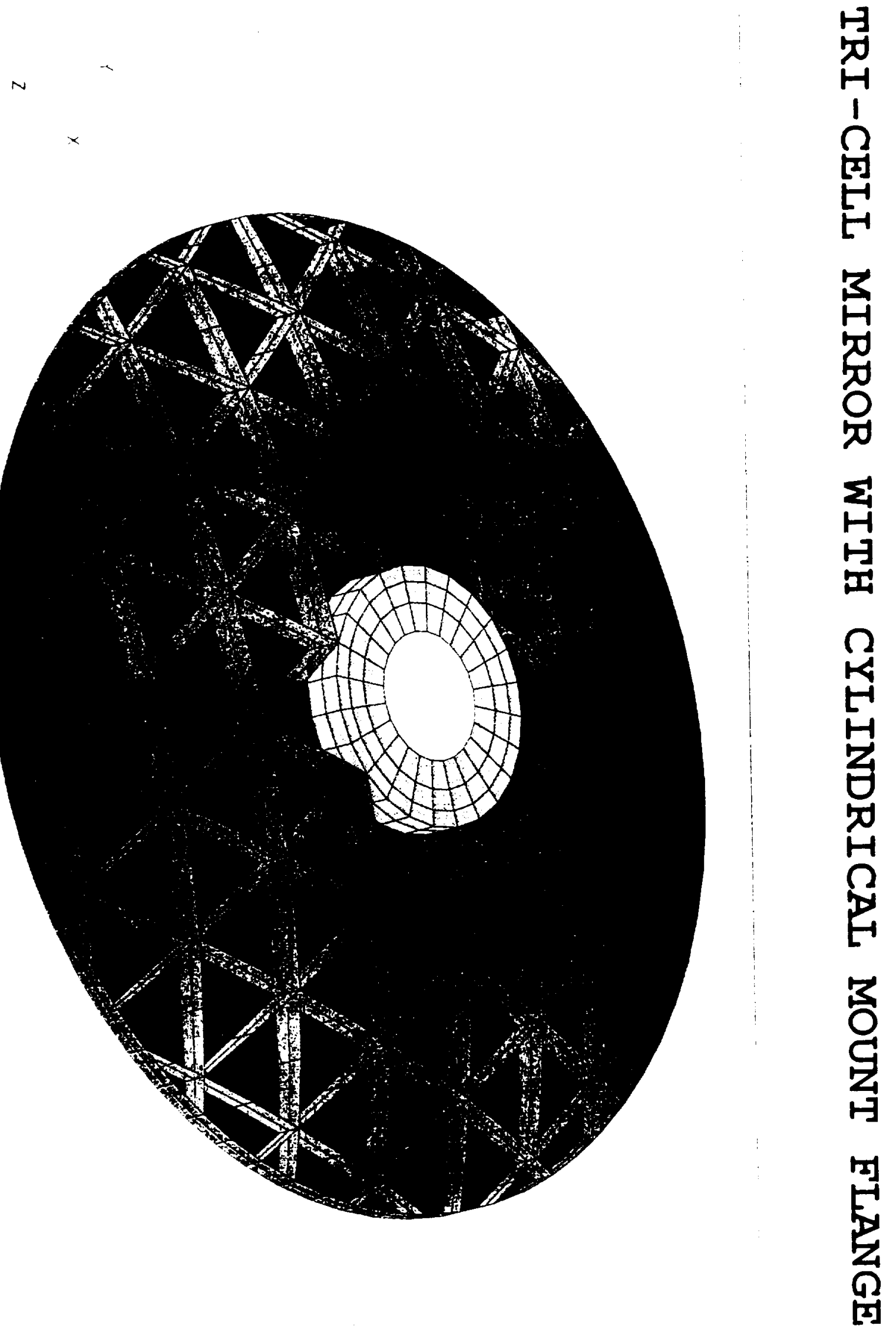

$\Omega \leq$ 


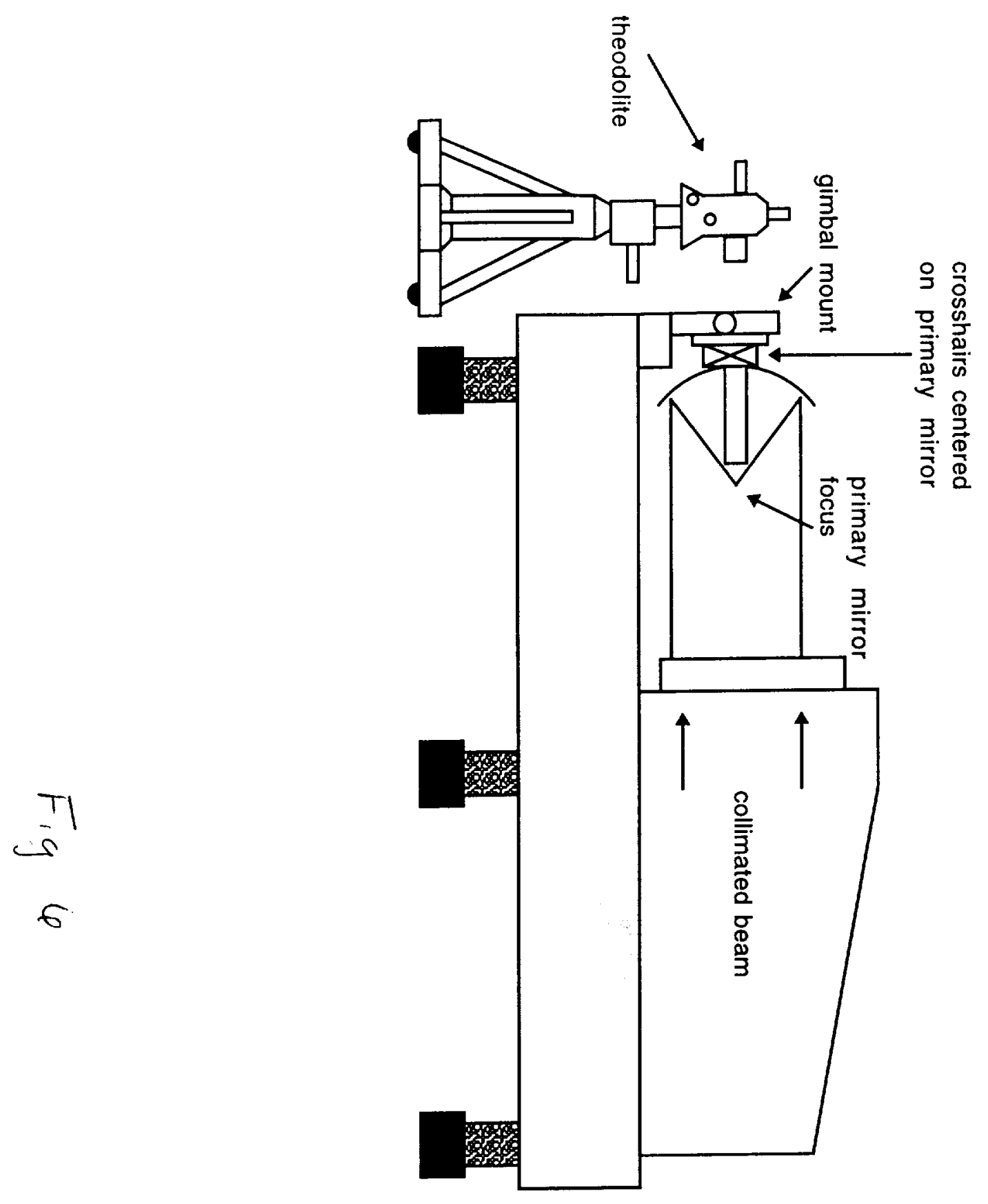



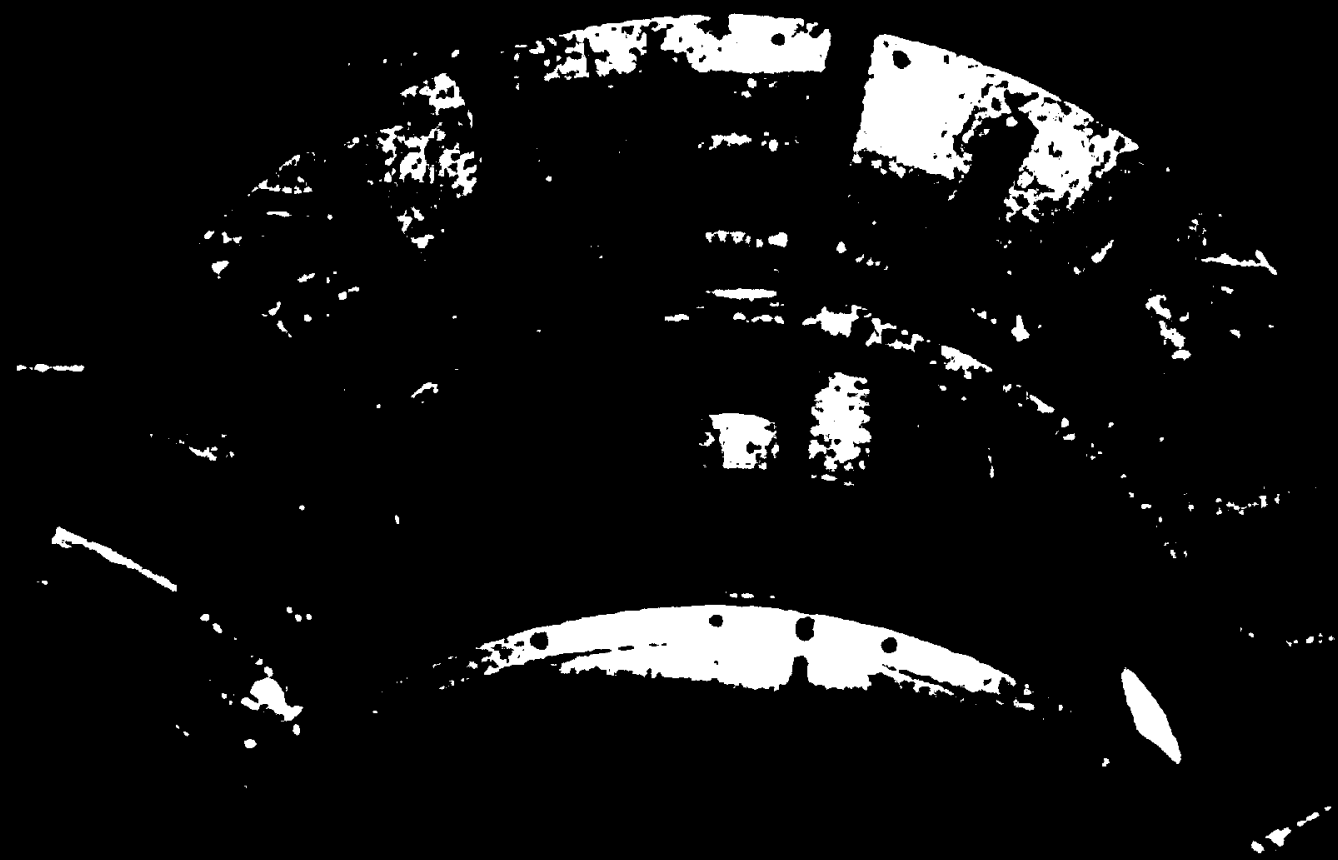

$\cdot$
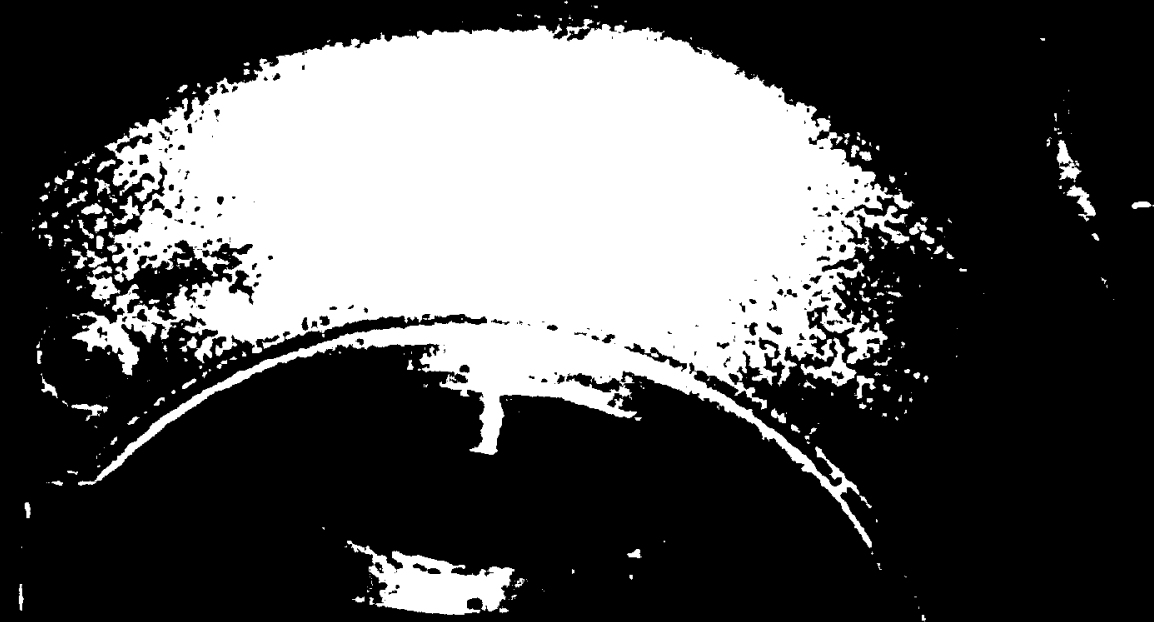

:
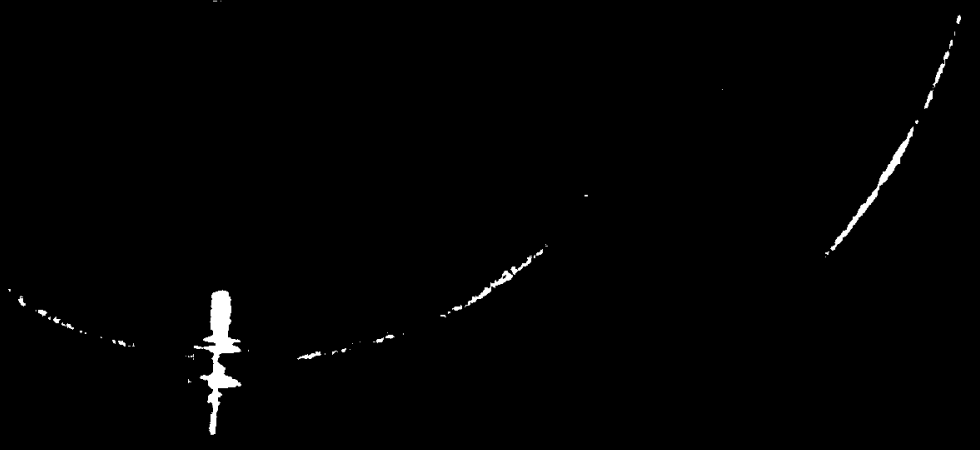


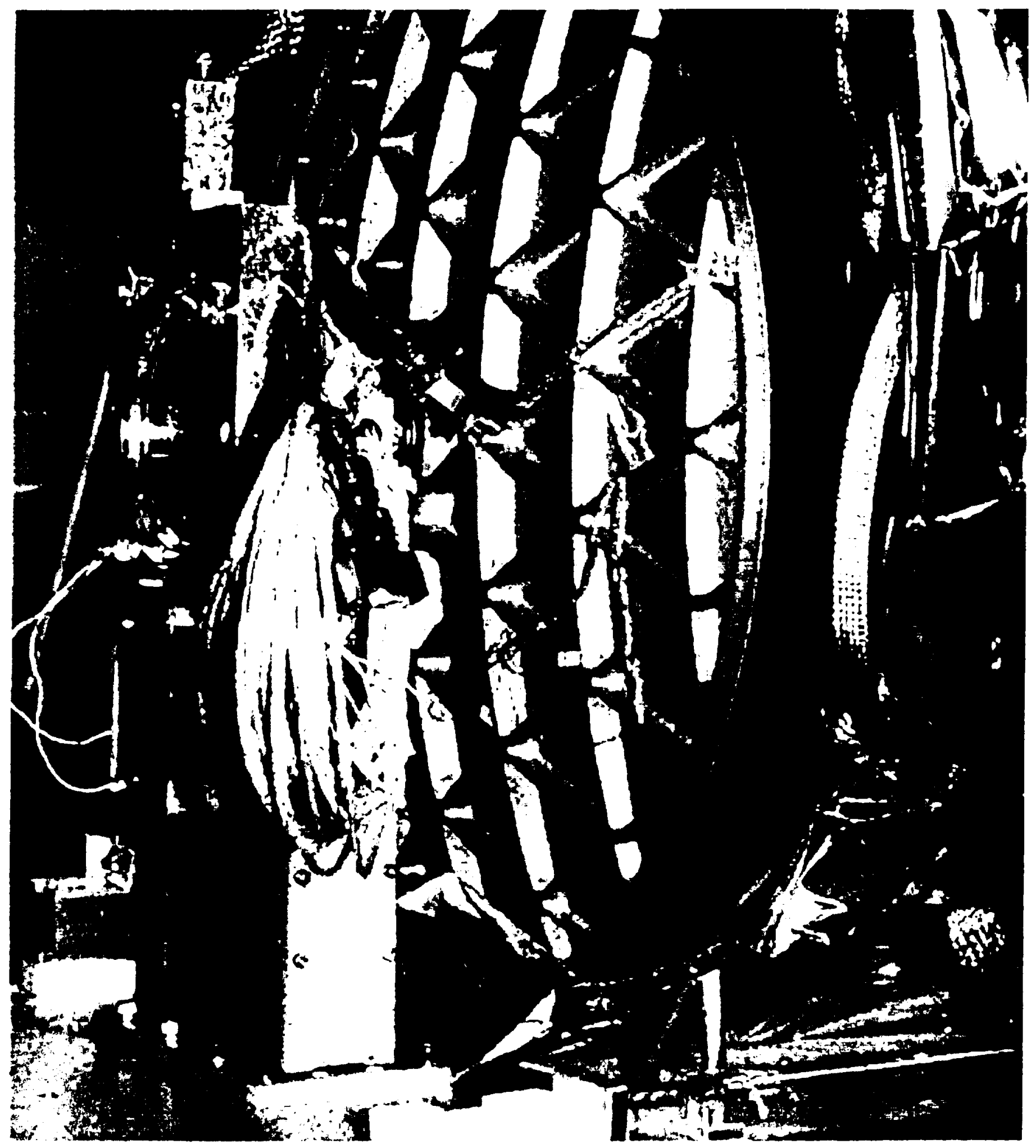

$F, g, c$ 


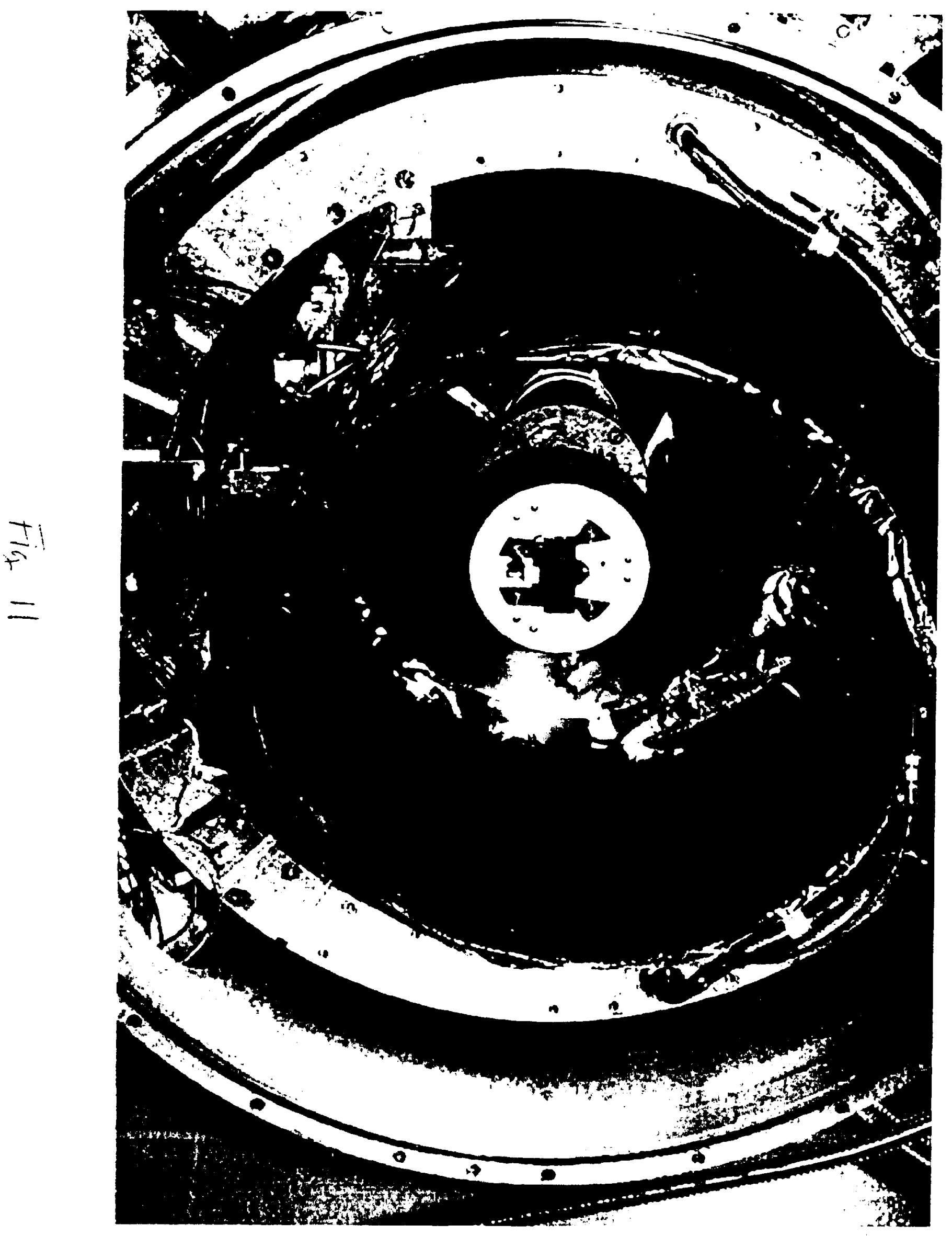




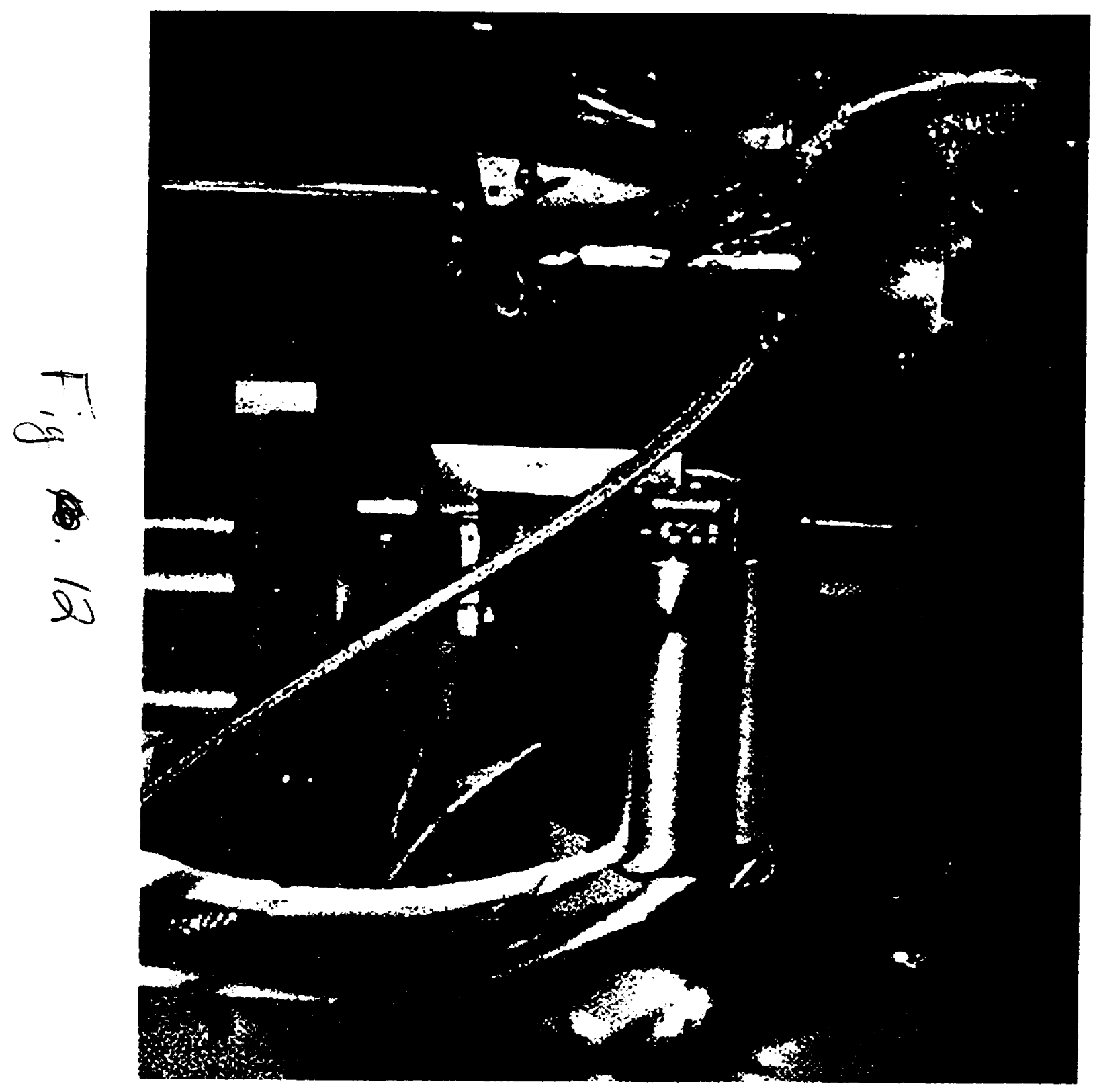




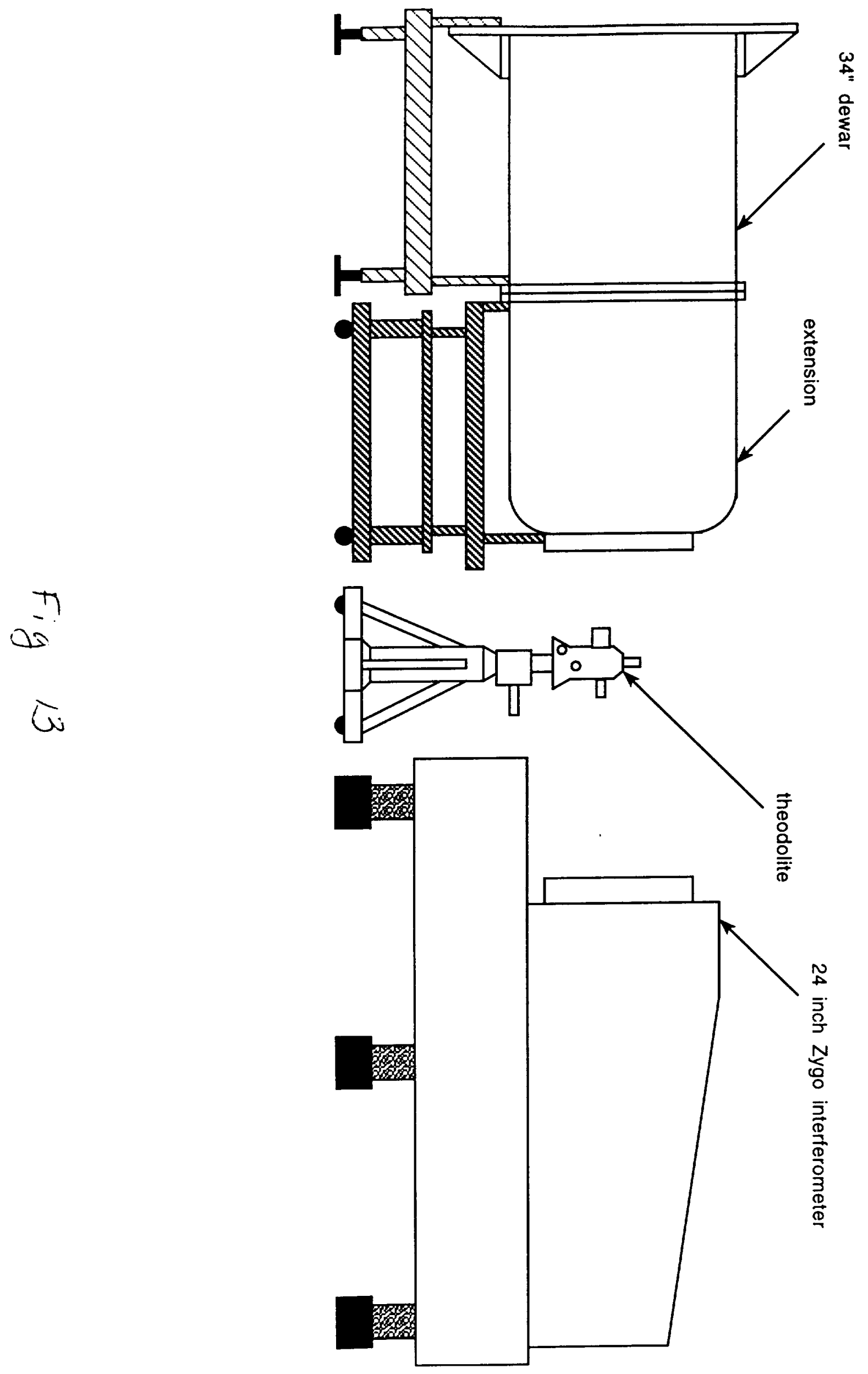

